Preprint HU-TFT-95-17

hep-ph/9503254

7 March 1995

\title{
FORMATION AND EVOLUTION OF QUARK-GLUON PLASMA AT RHIC AND LHC*
}

\author{
K.J. Eskola \\ Laboratory of High Energy Physics, \\ P.O. Box 9, 00014 University of Helsinki, Finland \\ email: kjeskola@ fltxa.helsinki.fi
}

\begin{abstract}
Initial conditions for quark-gluon plasma formation at $\tau=0.1 \mathrm{fm}$ are considered in lowest order perturbative QCD. Chemical composition, thermalization of the formed semihard quark-gluon system and especially implications of the new HERA parton distributions with the enhancement at small $x$ are studied. The plasma at $\tau=0.1 \mathrm{fm}$ is shown to be strongly gluon dominated both at RHIC and LHC, and a possibility for rapid thermalization of gluons at LHC is pointed out. Uncertainties in the calculations, particularly shadowing corrections to the parton distributions, are discussed. Free streaming and ideal hydro limits for the evolution of the gluonic plasma with the calculated minijet initial conditions are demonstrated, and a lower limit for final multiplicities obtained for the LHC nuclear collisions.
\end{abstract}

\footnotetext{
* Plenary talk at Quark Matter '95, Eleventh Conference on Ultra-Relativistic Nucleus-Nucleus Collisions, Monterey, CA, U.S.A., January 9-13, 1995.
} 


\title{
FORMATION AND EVOLUTION OF QUARK-GLUON PLASMA AT RHIC AND LHC
}

\author{
K. J. Eskola \\ Laboratory of High Energy Physics, P.O. Box 9, FIN-00014 University of Helsinki, \\ Finland
}

Initial conditions for quark-gluon plasma formation at $\tau=0.1 \mathrm{fm}$ are considered in lowest order perturbative QCD. Chemical composition, thermalization of the formed semihard quark-gluon system and especially implications of the new HERA parton distributions with the enhancement at small $x$ are studied. The plasma at $\tau=0.1 \mathrm{fm}$ is shown to be strongly gluon dominated both at RHIC and LHC, and a possibility for rapid thermalization of gluons at LHC is pointed out. Uncertainties in the calculations, particularly shadowing corrections to the parton distributions, are discussed. Free streaming and ideal hydro limits for the evolution of the gluonic plasma with the calculated minijet initial conditions are demonstrated, and a lower limit for final multiplicities obtained for the LHC nuclear collisions.

\section{INTRODUCTION}

During recent years, a lot of effort has been devoted to studying the space-time evolution of ultrarelativistic heavy ion collisions. Especially, from the point of view of quark-gluon plasma (QGP) formation, it is important to understand energy deposition and particle production mechanims in the central rapidity region of the collisions. Qualitatively these mechanisms can be divided into two categories: semihard and soft.

Semihard particle production is based on perturbative QCD (pQCD). The semihard gluons, quarks and antiquarks are produced in parton-parton collisions and they have transverse momenta $p_{T} \geq p_{0} \sim 1 \ldots 3 \mathrm{GeV}$. These semihard QCD quanta are often called minijets [1] because even in $p p(\bar{p})$ collisions they are not observable as individual jets below $p_{T} \sim 5 \mathrm{GeV}[2]$. However, even if minijets are not at all directly observable in nuclear collisions, they are expected to play a major role in the space-time evolution of central collisions at collider energies [3-7] Below the momentum transfer scale $\sim 1-2$ $\mathrm{GeV}$ pQCD is not expected to be reliable anymore, and modeling for soft particle production from beam jet fragmentation is needed. In heavy ion collisions this can be 
done e.g. in terms of strings as in hadron-hadron collisions [8], or in terms of a strong background color field decaying into particles through the Schwinger mechanism [9].

In the space-time evolution of an ultrarelativistic heavy ion collision the semihard and soft particle production mechanisms take place at different time scales. Semihard QCD quanta in the central rapidity region are typically formed at very early times, at $\tau \sim 1 / p_{T} \lesssim 1 / p_{0} \sim 0.1 \mathrm{fm}$, whereas the characteristic time scale for the soft processes (like the decay time of the background color field), is $\tau \sim 1 \mathrm{fm}$. In this sense, the minijet system is formed first and it will then serve as initial condition for the further evolution of the system. An example of this kind of modeling can be found e.g. in ref. [10].

In the central rapidity region the relative strength of the semihard and soft particle production mechanisms depends strongly on the cms energy of the heavy ion collision. The semihard part is expected to play a more important role with increasing energy, so that it is negligible at energies $\sqrt{s} \lesssim 20 A \mathrm{GeV}$, becomes relevant at the Brookhaven Relativistic Heavy Ion Collider (RHIC) energies $\sqrt{s} \sim 200 \mathrm{AGeV}$, and finally dominates at the CERN Large Hadron Collider (LHC) energies $\sqrt{s} \sim 5.5 A \mathrm{TeV}[3,4,6,11]$. This conclusion is based on the idea of multiple minijet production as the cause of the rapidly rising inelastic and total cross sections of $p p$ and $p \bar{p}$ collisions at energies $\sqrt{s} \gtrsim 200 \mathrm{GeV}$. A detailed discussion and further references can be found e.g. in ref [11].

Since the first ideas of the importance of the semihard processes in ultrarelativistic heavy ion collisions [3,5], a lot of inspiring work has been done on creating event generators like HIJING [6] and Parton Cascade Model (PCM) [7]. Naturally, the techniques of treating semihard processes in event generators like PYTHIA [12] for hadron-hadron collisions has been of great help in building up heavy ion event generators. In spite of the differences in initialization and in final state interactions, the semihard processes lie in the heart of generating events with e.g. HIJING and PCM at heavy ion collider energies. Therefore, to have a better control over the results from simulations, it is important to understand the basic processes as well as possible, and especially to study the uncertainties, like nuclear and hadronic shadowing of the parton distributions, higher order contributions to the cross sections, and the cut off scale $p_{0}$ used to give the division between semihard and soft physics.

In this talk, I will concentrate on the semihard parton production only. I will first estimate the initial conditions for early QGP formation. The main emphasis will be in consequences of the small- $x$ enhancement of the parton distributions observed in the HERA measurements, as studied in ref [13]. After considering the uncertainties in the pQCD calculation, I will discuss the evolution of the formed QGP with minijet initial conditions, and finally I will give an estimate for the lower limit of multiplicities for LHC nuclear collisions. 


\section{FORMATION OF QGP}

\subsection{Impact of the HERA measurements on minijet and $E_{T}$ production in $A A$ collisions}

The new deep inelastic ep-data from HERA [14] exhibit an increase in the structure function $F_{2}^{p}\left(x, Q^{2}\right)$ at small $x$ relative to the behavior implied by earlier data. Distribution function analyses using the new data have been carried out. These analyses, in particular, constrain the gluon distribution function, which is not directly measured. The estimates to be presented in this talk are computed with parton distributions D0', D-' and $\mathrm{H}$ by Martin, Roberts and Stirling (MRS) $[15,16]$. The corresponding gluon distributions for these sets at $Q=2 \mathrm{GeV}$ are shown in Fig. 1. At small $x$ the distributions shown behave as $x^{-\delta}$ with $\delta=0,-0.3$ and -0.5 , for D0', H and D-', correspondingly. In the global analysis by MRS, the set $\mathrm{H}$ follows from the best fit to the HERA data [16].

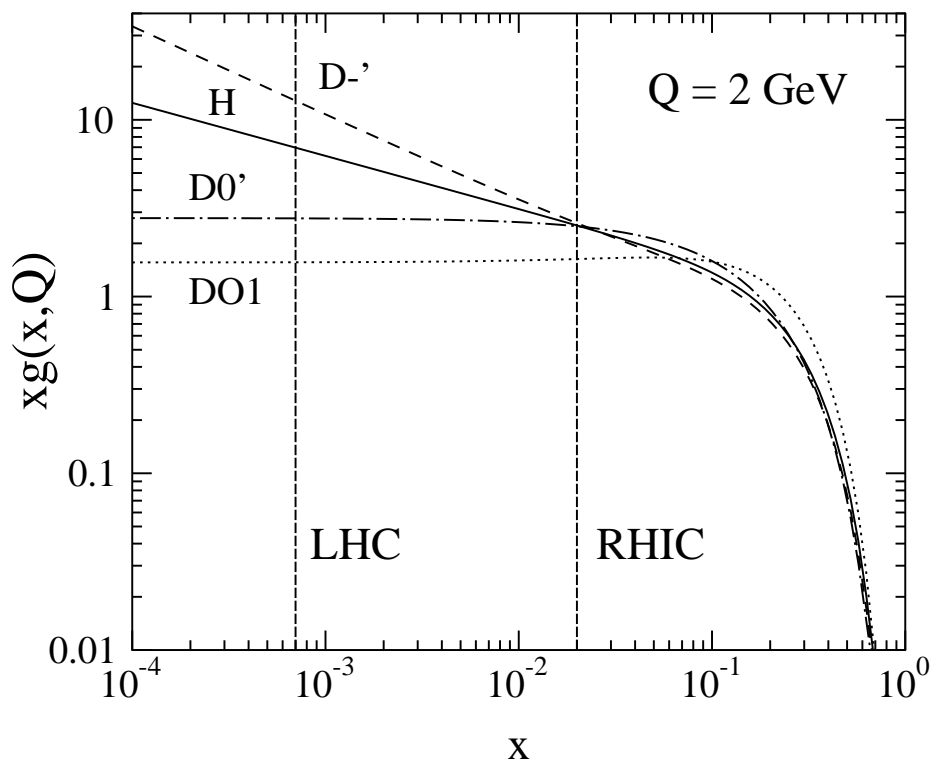

Fig. 1. Gluon distribution functions MRS D0', D-, and $\mathrm{H}[15,16]$ at the scale $Q=2$ $\mathrm{GeV}$. The old gluon distribution of Duke-Owens set 1 (DO1) [17] is shown for comparison. For the vertical lines, see the text.

For minijet production at high energies the consequences of the observed small- $x$ rise are obvious. Minijets falling into the mid-rapidity $y=0$ dominantly have transverse momenta $p_{T} \sim p_{0} \sim 2 \mathrm{GeV}$, and they mainly come from momentum fractions $x \sim$ $2 p_{0} / \sqrt{s}$. In Fig. 1, the vertical lines illustrate the typical value of $x$ for LHC and RHIC with $\sqrt{s}=200 \mathrm{GeV}$ and $\sqrt{s}=5500 \mathrm{GeV}$, correspondingly. Difference between the MRS distributions shown is relatively small at RHIC, whereas at LHC there is a sizable difference between the sets, and this is reflected into the minijet cross sections discussed below. 
When considering the number of produced semihard gluons, quarks and antiquarks, both in hadronic and nuclear collisions, the key quantity is the integrated (mini)jet cross section

$$
\sigma_{\text {jet }}\left(\sqrt{s}, p_{0}\right)=\int_{p_{0}^{2}}^{s / 4} d p_{T}^{2} d y_{1} d y_{2} \frac{1}{2} \frac{d \sigma_{\text {jet }}}{d p_{T}^{2} d y_{1} d y_{2}},
$$

where the inclusive differential jet cross section is given by

$$
\frac{d \sigma_{\text {jet }}}{d p_{T}^{2} d y_{1} d y_{2}}=K \sum_{\substack{i j k l=\\ q, \bar{q}, g}} x_{1} f_{i / p}\left(x_{1}, p_{T}^{2}\right) x_{2} f_{j / p}\left(x_{2}, p_{T}^{2}\right){\frac{d \hat{\sigma}^{i j \rightarrow k l}}{d \hat{t}}}^{i n}
$$

The momentum transfer in the process $i j \rightarrow k l$ is $p_{T}$, the fractional momenta of the partons $i$ and $j$ are $x_{1,2}$, and $y_{1,2}$ are the rapities of the partons $k$ and $l$. The calculation is only done to leading order $\left(\mathcal{O}\left(\alpha_{s}^{2}\right)\right)$. The higher order terms are in principle available from the calculation of S.D. Ellis-Kunszt-Soper (EKS) [18] but here these are simulated simply by a constant factor $K \sim 2$, which seems to be an acceptable approximation with a scale choice $Q=p_{T}[19]$.

In the eikonal formulation (see e.g. [11] and references therein) we can interpret $2 \sigma_{\text {jet }} / \sigma_{\text {in }}^{p p}$ as the average number of semihard partons with $p_{T} \geq p_{0}$ produced in an inelastic $p p$ collision. This ratio may become much larger than 2 , (see e.g. $\sigma_{\text {jet }}$ shown in fig. 1 in [13]) especially at LHC energies with small- $x$ enhanced gluon distributions, which indicates that one is effectively including minijet pairs not only from multiple (independent) semihard collisions but also contributions from the initial partonic flux, i.e. from the gluon ladders leading to the virtuality $Q \sim p_{T}$ [20]. However, in the central rapidity, the pairs should dominantly come from the semihard collisions.

In the first approximation, where all nuclear modifications to the parton densities $f_{i}$ are neglected, the average number of minijets produced in an $A A$ collision with an impact parameter $\mathbf{b}$ is obtained from

$$
\bar{N}_{A A}\left(\mathbf{b}, \sqrt{s}, p_{0}\right)=T_{A A}(\mathbf{b}) 2 \sigma_{\text {jet }}\left(\sqrt{s}, p_{0}\right)
$$

where $T_{A A}(\mathbf{b})$ is the standard nuclear overlap function. Here we will need only $T_{A A}(\mathbf{0}) \approx$ $A^{2} /\left(\pi R_{A}^{2}\right) \approx 32 / \mathrm{mb}$ for $A=P b$. Notice that there are two reasons for large $\bar{N}_{A A}$ for heavy nuclei: the overlap function scales as $\sim A^{4 / 3}$ and the perturbative cross section $\sigma_{\text {jet }}$ becomes large at high energies.

The results for $\sigma_{\text {jet }}$ integrated over all rapidities can be found in [13], but what is of greater interest for us here, are the numbers of minijets falling into the central rapidity unit $|y| \leq 0.5$. By making this cut in Eq. (1) (as will be done in Eq. (4)) and by careful bookkeeping of the flavors which fall into this rapidity window, we find out an estimate for the chemical composition of the produced minijets at LHC and at RHIC. This is shown in Table 1.

We make two observations: First, as expected, the small- $x$ rise of the parton distributions does not affect much the numbers for RHIC, but inreases considerably the 


\begin{tabular}{lrrrr}
\hline Set & total & $g$ & $q$ & $\bar{q}$ \\
\hline DO1 & 776 & 640 & 72 & 64 \\
& 134 & 100 & 21 & 13 \\
& & & & \\
D0' & 1510 & 1250 & 136 & 128 \\
& 207 & 162 & 27 & 18 \\
& & & & \\
H & 3250 & 2710 & 276 & 266 \\
& 200 & 157 & 26 & 17 \\
& & & & \\
D-' & 5980 & 5220 & 385 & 373 \\
& 200 & 157 & 26 & 17 \\
\hline
\end{tabular}

Table. 1. Values of $\bar{N}_{A A}\left(\mathbf{0}, \sqrt{s}, p_{0}\right)$ calculated for $p_{0}=2 \mathrm{GeV}, \sqrt{s}=200 \mathrm{GeV}$, $5500 \mathrm{GeV}$ and for the three parton distributions considered in the text. The upper numbers are for LHC and the lower ones for RHIC. The results with Duke-Owens set 1 distributions (DO1) are shown for comparison. An overall factor $K=2$ but no shadowing is included. Both the total as well as the contribution from gluons, quarks and antiquarks is given.

numbers for LHC. Secondly, the formed minijet plasma is obviously strongly gluon dominated: about $80 \%$ of the produced partons are gluons. At this point, one should bear in mind that the figures above are only the lowest order estimates, and the number of partons is well defined. However, when considering higher order terms in the jet cross section, one encounters collinear singularities in the case of three-jet final states, and, without a definition of a jet size $R$ in the $(y, \phi)$-plane it is not clear when the partons should be counted as separate jets and when as one jet [18]. In general, radiative effects would tend to increase the gluon dominance of the formed minijet plasma.

Let us then consider perturbatively the transverse energy in the central rapidity region of $A A$ collisions. This can be estimated (see [4] for details) in terms of the first $p_{T}$ moment

$$
\sigma_{\text {jet }}\left(\sqrt{s}, p_{0}\right)\left\langle E_{T}\right\rangle=\int_{p_{0}} d p_{T} d y_{1} d y_{2} \frac{d \phi}{2 \pi} \frac{1}{2} \frac{d \sigma_{\text {jet }}}{d p_{T} d y_{1} d y_{2}}\left(\epsilon_{1}+\epsilon_{2}\right) p_{T},
$$

where the rapidity cut is made by the functions $\epsilon_{k}: \epsilon_{k}=\epsilon\left(p_{T}, y_{k}\right)=1$ if the parton $k$ falls inside the region of our interest and $=0$ otherwise. To illustrate the $p_{0}$ dependence, $\sigma_{\text {jet }}\left\langle E_{T}\right\rangle$ is plotted as a function of $p_{0}$ in Fig. 2. Note again that no shadowing is included in the calculation. The composition into contributions from gluons, quarks and antiquarks with $p_{0}=2 \mathrm{GeV}$ is shown in Table 2. The estimate for the total initial 
$E_{T}$ in one unit of $y$ near $y=0$ is then

$$
\begin{aligned}
& \bar{E}_{T}^{P b P b}(\mathbf{0})\left(\sqrt{s}, p_{0},|y| \leq 0.5\right)=T_{P b P b}(\mathbf{0}) \sigma_{\text {jet }}\left(\sqrt{s}, p_{0}\right)\left\langle E_{T}\right\rangle \\
& =3200(387) \mathrm{GeV} \quad \text { DO1 }: x g(x) \sim \text { const, } \\
& =5440(563) \mathrm{GeV} \quad \mathrm{D}^{\prime}: x g(x) \sim \text { const, } \\
& =10300 \quad(547) \mathrm{GeV} \quad \mathrm{H} \quad: x g(x) \sim x^{-0.3} \text {, } \\
& =17600 \quad(538) \mathrm{GeV} \quad \mathrm{D}-^{\prime}: x g(x) \sim x^{-0.5} \text {. }
\end{aligned}
$$

where the numbers are for LHC (RHIC), correspondingly.

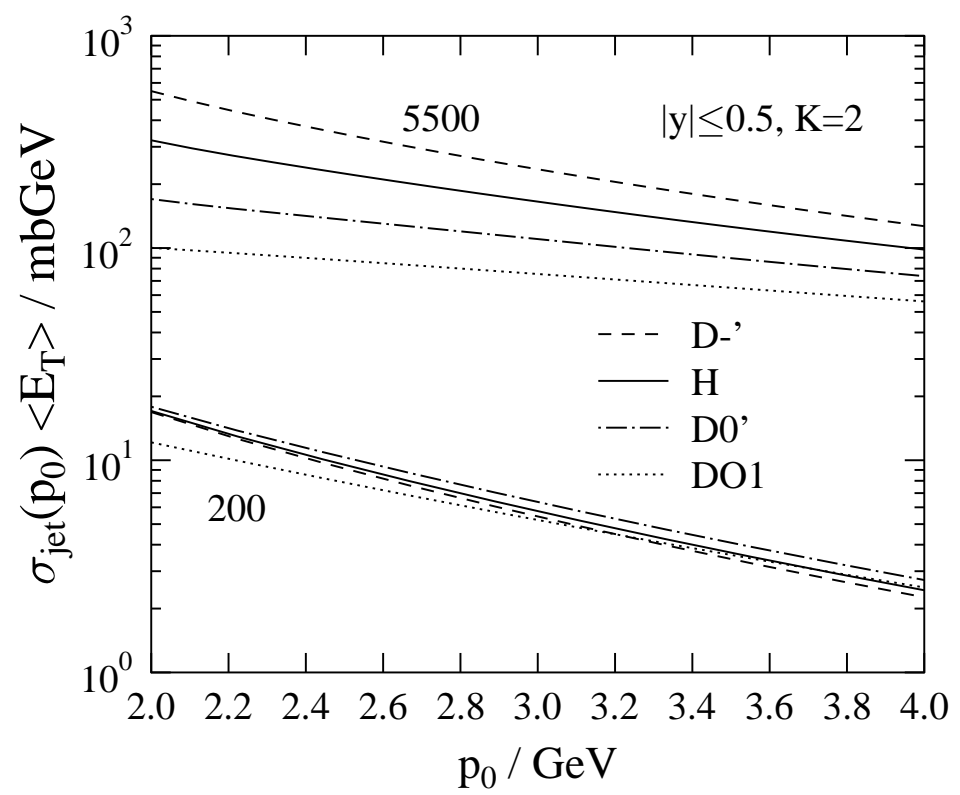

Fig. 2. The first $p_{T}$ moment $\sigma_{\text {jet }}\left(\sqrt{s}, p_{0}\right)\left\langle E_{T}\right\rangle$ from Eq. (4) with the sets of distribution functions marked on the figure and for $\sqrt{s}=200$ and $5500 \mathrm{GeV}$ [13].

Again, the increase in $E_{T}$ associated with the small- $x$ increase is significant for LHC. However, this $E_{T}$ is the final $E_{T}$ only if no further interactions take place in the system. The largest decrease in $E_{T}$ is obtained if the system thermalizes and an ideal hydro flow is generated. Another interesting point is that in spite of the apparently large values of $E_{T}$ in Eq. (5), at most only about $1.5 \%$ of the total energy is stopped perturbatively.

Since the longitudinal size of the comoving volume is $\Delta z=\tau \Delta y$ and $\tau_{i} \sim 1 / p_{0}=$ $0.1 \mathrm{fm}$ the obtainable energy densities can be estimated with the Bjorken formula [21] as $\epsilon\left(\tau_{i}\right)=\left\langle E_{T}^{A}(|y|<0.5)\right\rangle p_{0} / \pi R_{A}^{2}$. Already without the small- $x$ enhancement of the parton distributions, i.e. for distributions like MRSD0', the energy densities $\epsilon\left(\tau_{i}\right)$ become large as compared to the critical density $\epsilon_{c} \sim 1 \ldots 3 \mathrm{GeVfm}^{-3}$ associated with the deconfinement phase transition. For MRSD0' we get $\epsilon\left(\tau_{i}\right) \sim 410$ (43) $\mathrm{GeVfm}^{-3}$ for LHC (RHIC). An interesting observation is that for distributions like MRSD0' at high enough energies the initial energy density does not depend strongly of $p_{0}$. This is 


\begin{tabular}{lrrrr}
\hline Set & $\sigma_{\text {jet }}\left\langle E_{T}\right\rangle$ & $g$ & $q$ & $\bar{q}$ \\
\hline DO1 & 100 & 84.5 & 8.29 & 7.41 \\
& 12.1 & 9.16 & 1.87 & 1.11 \\
& & & & \\
D0' & 170 & 142 & 14.5 & 13.6 \\
& 17.6 & 14.0 & 2.12 & 1.50 \\
& & & & \\
H & 322 & 270 & 26.7 & 25.5 \\
& 17.1 & 13.3 & 2.30 & 1.47 \\
& & & & \\
D-' & 549 & 479 & 35.8 & 34.5 \\
& 16.8 & 13.2 & 2.24 & 1.40 \\
\hline
\end{tabular}

Table. 2. As Table 1 , but for $\sigma_{\text {jet }}\left(p_{0}\right)\left\langle E_{T}\right\rangle$ in units of mbGeV with $|y| \leq 0.5, \sqrt{s}=$ $200 \mathrm{GeV}, 5500 \mathrm{GeV}$ and $p_{0}=2 \mathrm{GeV}$.

because of the scalings: $\sigma_{\text {jet }}\left(p_{0}\right) \sim p_{0}^{-2}, \sigma_{\text {jet }}\left(p_{0}\right)\left\langle E_{T}\right\rangle \sim p_{0}^{-1}$, and the $p_{0}$-dependence in $\epsilon\left(\tau_{i}\right)$ is canceled out. With the small- $x$ enhancement of the parton distributions, the results depend more on $p_{0}$, as illustrated in Fig. 2.

About the approximations done, one should note that the transit times of the colliding nuclei were neglected. In $A A$ collisions with $A \sim 200$ at RHIC the transit time is about $0.2 \mathrm{fm}$, which will reduce the estimate for the maximum energy density by a factor $\sim 2[10]$. For LHC, the transit time is negligible as compared to $1 / p_{0}=0.1 \mathrm{fm}$, but nuclear shadowing may reduce the estimate at least by a similar factor (see e.g. [34]).

\subsection{Chemical equilibrium at $\tau=0.1 \mathrm{fm}$ ?}

The lowest order pQCD results of [13] described in the previous chapter show that the minijet-gluons dominate the early QGP. Let us now study how far from chemical equilibrium we actually are initially at $\tau \sim 0.1 \mathrm{fm}$.

For an ideal gas of massless gluons, quarks and antiquarks, we have the following ratios at zero chemical potential:

$$
\left.\frac{n_{g}}{n_{q}+n_{\bar{q}}}\right|_{\text {ideal }}=\frac{16}{9 N_{f}} \leq 0.9,\left.\quad \frac{\epsilon_{g}}{\epsilon_{q}+\epsilon_{\bar{q}}}\right|_{\text {ideal }}=\frac{32}{21 N_{f}} \leq 0.8, \quad N_{f} \geq 2
$$

From the previous lowest order pQCD results, we can extract the ratios as

$$
\left.\frac{n_{g}}{n_{q}+n_{\bar{q}}}\right|_{\mathrm{pQCD}} \sim 3.7(\mathrm{RHIC}) \ldots . .5(\mathrm{LHC}),\left.\quad \frac{\epsilon_{g}}{\epsilon_{q}+\epsilon_{\bar{q}}}\right|_{\mathrm{pQCD}} \sim 3.5(\mathrm{RHIC}) \ldots . .5(\mathrm{LHC})
$$

The conclusion is the same from both number and energy densities and for both RHIC and LHC: the pQCD ratios are far from the equilibrium ones. Many more quarks and antiquarks would be needed for complete chemical equilibrium. 
Then, an interesting question is that if we consider gluons only, can they be in chemical equilibrium, i.e. thermalized at $\tau \sim 0.1 \mathrm{fm}$ ? In this case it would mean that the gluon number density is close to the thermal density obtained by assuming that all the initial energy density, $\epsilon_{g}\left(\tau_{i}\right)$, is fully thermalized. Therefore, for LHC, we compare energy/gluon computed in two ways:

i) take the previous pQCD results from tables 1 and 2 to get $\bar{E}_{T, g}^{P b P b}$ and $\bar{N}_{P b P b}^{g}$ with $p_{0}=2 \mathrm{GeV},|y| \leq 0.5$.

ii) take the pQCD estimate for $\bar{E}_{T, g}^{P b P}$, convert this into equilibrium $\left(\mu_{g}=0\right)$ energy density $\epsilon_{g}\left(\tau_{i}\right)=3 a T_{i}^{4}$, where $a=16 \pi^{2} / 90$, solve for $T_{i}$, and get energy/gluon as $\epsilon_{g}\left(\tau_{i}\right) / n_{g}\left(\tau_{i}\right) \approx 2.7 T_{i}$. For LHC, the results for different parton distributions are

$$
\begin{aligned}
& E / \text { gluon }=4.1 \mathrm{GeV} \quad 2.7 T_{i}=2.0 \mathrm{GeV} \quad \text { DO1 } \\
& =3.6 \mathrm{GeV} \quad=2.2 \mathrm{GeV} \quad \mathrm{D0}^{\prime} \text {, } \\
& =3.2 \mathrm{GeV} \quad=2.6 \mathrm{GeV} \quad \mathrm{H} \text {, } \\
& =2.9 \mathrm{GeV} \quad=3.0 \mathrm{GeV} \quad \mathrm{D}^{\prime} \text {. }
\end{aligned}
$$

For the distributions with $x g(x) \sim$ const the thermalization of gluons requires a degradation of their average energy by collisions. For RHIC this is also the case [22]. With the HERA parton distributions the secondary interactions are only needed for changing the directions of momenta to make the distribution uniform. The conclusion is that the assumption of fast initial thermalization seems reasonable at LHC when new HERA parton densities are used.

\subsection{Uncertainties: $K, p_{0}$ and shadowing}

In the previous results for the jet cross sections there are three main uncertainties: the $K$-factor, the momentum cut-off $p_{0}$ and shadowing of the parton distributions.

The calculations were done only to the lowest order (LO) pQCD, and a factor $K=2$ was used together with a scale choice $Q=p_{T}$ to simulate the contributions from higher orders of $\alpha_{s}$. Here one should note that a ' $K$-factor' always depends on the scale choice; it can even be removed by choosing the scale differently. The Born level jet cross section does not depend on the jet size $R$ in the $(y, \phi)$-plane, while the next-to-leading order (NLO) calculation [18] does, and the experimentally measured jet cross sections do. Therefore, the $K$-factor always depends on the jet size, regardless of whether $K$ is defined by comparing the LO results to the inclusive jet data [23] or to the LO+NLO calculation. Naturally, $K$ also depends on the parton distributions and may also depend on the cms energy.

Although I am not discussing the inclusive jet production here but, rather, considering more semi-inclusive quantities, (i.e. all minijets and their transverse energy in the central rapidity unit), I show in Fig. 3 the inclusive 1-jet distribution from [19] in $p p$ collisions at $\sqrt{s}=200 \mathrm{GeV}$ with $Q=p_{T}$ and $R=1$. This shows that with this scale 


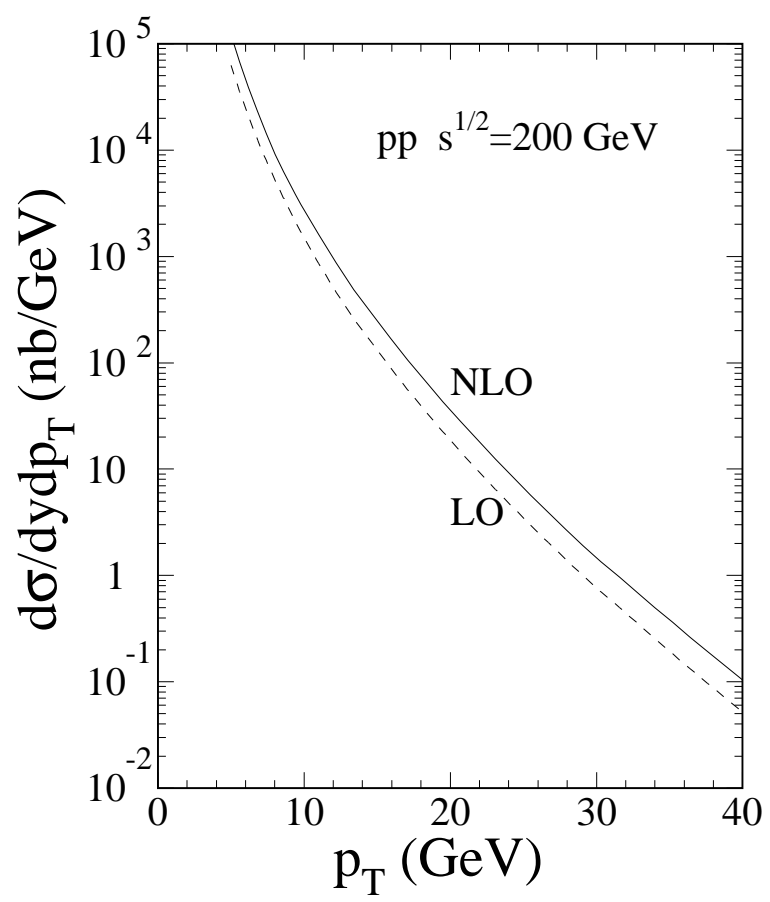

Fig. 3. The inclusive one-jet cross section $d \sigma / d p_{T} d y$ vs. $p_{T}$ at $y=0$ in $p p$ collisions at $\sqrt{s}=200 \mathrm{GeV}$, as predicted by the NLO (solid) and LO(dashed) calculations with $Q=p_{T}$. The MRSD-' parton distributions were used. For the NLO calculation $R=1.0$. The figure is from ref. [19], and the calculation was done by using the program by EKS [18].

choice, it is a good approximation to have constant $K \sim 2$ to simulate the NLO terms in the jet cross sections.

When the NLO contributions to the jet cross sections are included, the dependence on the scale choice should be weaker. This has been nicely demonstrated in [18] (see also [19]), and it works well for jets with $p_{T} \gtrsim 10 \mathrm{GeV}$. However, when approaching the minijet region $p_{T} \sim p_{0} \sim 2 \mathrm{GeV}$, the convergence of the perturbation series becomes worse, and one would eventually need even higher order terms in the jet cross sections in order to improve the determination of the scale choice. This does not mean that the LO minijet results would be incorrect, it simply means that it may be very difficult to pin down the accuracy of the minijet calculation below a factor 2. As an example of this, we see in Fig. 3 that at least down to $p_{T}=5 \mathrm{GeV}$ the jet NLO calculation does not exhibit any striking behavior as compared to the LO result, even though at this low $p_{T}$ the NLO result depends on the scale choice practically as much as the LO does. However, it is clear that this problem needs further studies.

In the approach considered here, the parameter $p_{0}$ defines a division into semihard and soft physics. Therefore, $p_{0}$ is a phenomenological parameter, and data on multiplicities and cross sections are needed to determine $p_{0}$. In the eikonal approach for the $p \bar{p}(p)$ collisions, the cross section $\sigma_{\text {soft }}$ for processes with $p_{T}<p_{0}$ is modeled in together with $\sigma_{\text {jet }}\left(p_{0}\right)$. Through the condition that experimentally measured $p \bar{p}(p)$ total cross sections are reproduced, the value of $\sigma_{\text {jet }}\left(p_{0}\right)$ then depends on the choice for $\sigma_{\text {soft }}[1,11]$. 
In principle, in determination of $p_{0}$, one should also compare the final multiplicities as was done in [11]. Rather than doing a complete analysis like this, I have shown the results from [13] as a function of $p_{0}$. However, a reanalysis on determination of $p_{0}$ with the new HERA parton distributions should be done, and the possible $A$ dependence of $p_{0}$ should be studied in more detail.

Related to the cut-off $p_{0}$, I note the following. Part of the higher order contributions come from $2 \rightarrow 2+(n \geq 1)$ processes. At the tree level, these have been studied recently by E. Shuryak and Li Xiong [24], based on the matrix elements in [25]. In this treatment, one is faced with the jet resolution problem mentioned before, and with cut-off $s_{i j}>s_{0} \sim 4 \mathrm{GeV}^{2}$ for each pair $i j$, the higher order tree graphs seem to become dominant over the $2 \rightarrow 2$ processes at LHC energies [24]. Again, this shows that the actual number of gluons depends on the resolution, and is connected with color screening as well. Certainly, this interesting problem needs further studies, especially for LHC energies.

Let us then come to the uncertainty due to modifications of the parton distributions. Since here we are mostly interested in the small- $x$ phenomena, I will only consider shadowing.

Shadowing in a proton means a depletion or a saturation in the gluon and sea quark distributions at $x \lesssim 0.01$. For the distributions behaving as $x g \sim x^{-\delta}$, at sufficiently small values of $x$ and/or $Q$ the partons start to overlap spatially, and a reduction in the parton density results due to the occurring recombinations. Corrections from the gluon fusions to the Dokshitzer-Gribov-Lipatov-Altarelli-Parisi (DGLAP) scale evolution [26] at small $x$ have been calculated in the leading double logarithm approximation by Gribov-Levin-Ryskin and Mueller-Qiu (GLRMQ) [27]. In the semiclassical approximation it has been shown that after correcting the evolution of the steeply rising gluon distributions $\sim x^{-\delta}$, they behave at asymptotically small $x$ as $x g \sim$ const [28]. At RHIC energies, the shadowing corrections in proton can obviously be neglected but for LHC energies they should be taken into account, at least with the new HERA parton distributions.

There are two sources of gluon recombinations causing the shadowing in a proton: the two fusing gluon ladders, which couple four gluons to two gluons, can arise either from independent constituents of a proton or from the same one, as discussed in [27-29]. The HERA data for the structure function $F_{2}^{p}$ would seem to favor the first possibility; the latter source may be ruled out as too strong a fusion since no saturation in $F_{2}$ is seen so far. However, since the sea quarks are emitted from the gluons they carry smaller $x$ than the mother gluons, and the saturation of the quark distributions should show up at somewhat smaller $x$ than that of gluons. It would be important to understand this better, since nuclear gluon shadowing arises perturbatively from the same sources as in the proton shadowing, with possibly different relative strengths [30].

Nuclear effects in the parton distributions in general have been widely studied during the recent years (see e.g. [31] for further references). However, a unified description through the whole $x$-range is very difficult to make, due to the different origin of the 
effects at different ranges of $x$. From the point of view of phenomena discussed in this talk, shadowing as the small- $x$ effect is the most important one. Notice also that an interesting theoretical idea for finding out the nuclear gluon densities at small $x$ was discussed in this conference by R. Venugopalan [32].

Shadowing in a nucleus means a depletion of the nuclear parton densities relative to the parton densities in a proton: $f_{i}^{A}\left(x, Q^{2}\right)<A f_{i}^{p}\left(x, Q^{2}\right)$ at $x \lesssim 0.1$. Unlike shadowing in proton, nuclear shadowing of the structure function $F_{2}^{A}$ is experimentally clearly observed in deep inelastic scattering [33], and it seems to be fairly independent of the scale $Q$. Although measured in fixed target experiments, nuclear shadowing gets a simple qualitative explanation in the infinite momentum frame in terms of overlapping partons. If the longitudinal wavelength of a parton, $\sim 1 /(x p)$, exceeds the contracted size of the nucleons (or the inter-nucleon distance) in the nucleus, $\sim 2 r_{n} m_{n} / p$, shadowing should show up at $x \lesssim 1 /\left(2 m_{n} r_{n}\right) \sim 0.1$. According to the data this seems to be the case. Also, at sufficiently small $x$ the partons from all nucleons at the same transverse location will overlap, and a saturation of $f_{i}^{A} / A f_{i}^{p}$ is expected, at least when $f_{i}^{p}$ starts saturating (see e.g. [30]).

Now, in the minijet calculation with the latest HERA parton distributions, inclusion of shadowing is not a trivial matter. The nuclear shadowing effects are often simulated by simply multiplying the unshadowed parton distribution in a proton by a $Q$-independent ratio, extracted from the data [33] for $F_{2}^{A} / A F_{2}^{p}$. While this is presumably a rather good approximation for the sea quarks, it may not at all be the right thing to do with the gluons. Even when neglecting the shadowing in a proton - which should be a reasonable approximation at the RHIC energies - the ratio $x g_{A} / A x g_{p}$ may be more strongly $Q$-dependent than that of the sea quarks. This was demonstrated in [34]. Besides the $Q$-dependence, one should also make sure that the valence quark and momentum sum rules are fulfilled for the nuclear parton distributions as well.

At LHC energies the problem is more complicated since the gluon shadowing in a proton must not be neglected. Especially, with the rapidly rising gluon distributions, the gluon density in a nucleus at small $x$ cannot be reliably approximated by simply multiplying by $F_{2}^{A} / A F_{2}^{p}$ since $x g_{p}$ should be shadowed as well. With the absence of a direct measurement for the gluon densities, the nuclear gluon shadowing can at the moment only be modeled in theoretically. By using the same perturbative mechanism to generate gluon fusions as described in the proton case above, nuclear gluon shadowing was studied in [30]. These results have not been implemented yet into the minijet calculation, but the procedure is clear: After extracting the nuclear parton distributions at some scale $Q$ as in [30] and as in [34], one should then perform the full DGLAPGLRMQ evolution in the case of nucleus. This work is in progress [35]. To get a first feeling of the order of magnitude of the shadowing effects, I refer to figs 5 and 7 of [34]. 


\section{EVOLUTION OF THE QGP}

Let us then briefly consider the early evolution of the QGP in a one-dimensional longitudinal scaling expansion with boost invariant initial conditions [21]. The initial conditions are given by the system of formed semihard gluons, quarks and antiquarks at $\tau \sim 0.1 \mathrm{fm}$. As mentioned in the Introduction and as shown in [10], the soft processes are expected to be important for the evolution of the energy density $\epsilon(\tau)$ up to RHIC energies but to be negligible at LHC energies (see e.g. fig. 2 of [10]). Since in this talk I am only considering the semihard contribution, let me now comment on the evolution of the QGP at LHC energies only.

The QGP at $\tau=0.1 \mathrm{fm}$ is strongly gluon dominated. Let us therefore simplify the discussion by neglecting the fermions. Furthermore, as demonstrated before, with the small- $x$ enhanced gluons it is reasonable to approximate the gluons to be fully thermalized at $\tau=0.1 \mathrm{fm}$. Then, the evolution of the energy density $\epsilon_{g}(\tau)$ of the gluon plasma is governed by the equation [21]

$$
\frac{d \epsilon_{g}}{d \tau}+\frac{\epsilon_{g}+P_{g}}{\tau}=0
$$

where $P_{g}$ is the gluonic pressure. If viscosity and Ohmic heating effects are taken into account, they would appear on the right-hand side of the equation above. These have been studied e.g. in [10] in connection with particle production from the decaying chromo-electric background field. However, for the discussion below these effects are not relevant.

Now, there are two extreme cases for the evolution of the gluon plasma:

i) free streaming: $P_{g}=0 \Rightarrow \epsilon_{g} \sim \tau^{-1}$

ii) ideal hydro: $P_{g}=\epsilon / 3 \Rightarrow \epsilon_{g} \sim \tau^{-4 / 3}$

The assumption of full thermalization of glue is relevant only for the latter case. If viscosity was included, it could at most reduce the $P d V$ work to zero, so the first case is clearly an upper bound for the evolution of $\epsilon_{g}$ of a purely gluonic plasma (GP). In Fig. 4 the evolution of $\epsilon_{g}$ is plotted as a function of $\tau$ in the two extreme cases mentioned above. From the figure we see that if the deconfinement phase transition starts at $\epsilon_{g} \sim 1-2 \mathrm{GeV} \mathrm{fm}^{-3}$, even the minimum life time of the GP at LHC is $\sim 10 \mathrm{fm}$.

\subsection{Multiplicities at LHC - lower limit}

As a final topic, let me then estimate the multiplicities obtainable at LHC. The expected charged multiplicity is a crucial variable for the planning of experiments [36] and the various theoretical predictions lie roughly in the range 1500-8000.

Certainly, the free streaming case above would give us an upper limit for the multiplicity, $\sim \bar{E}_{T}^{P b P b}(|y| \leq 0.5) / 0.5 \mathrm{GeV}$. This would correspond to having no secondary interactions after the initial collision. In practice, this bound as such is of no realistic use, since the final state interactions are practically inevitable in this dense systems. The lower limit of multiplicity is obtained by considering only the gluons in the ideal hydro case (solid line in Fig. 4), where the system is fully thermalized at $\tau=0.1 \mathrm{fm}$ 


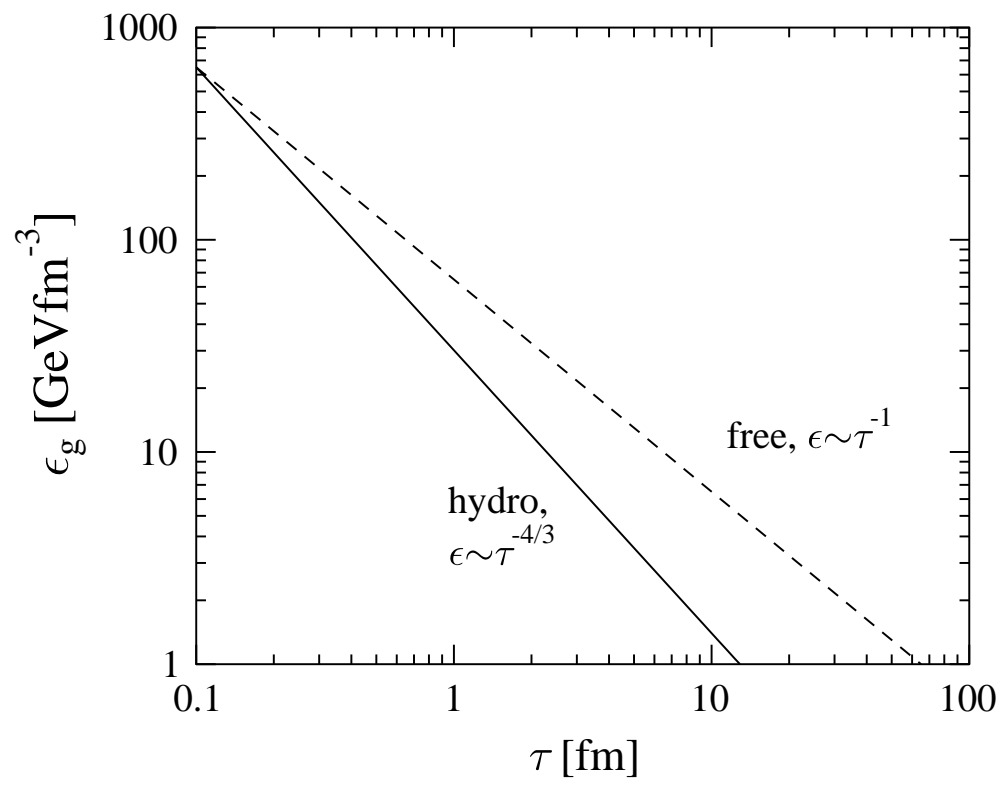

Fig. 4. The two extreme cases of the evolution of the energy density $\epsilon_{g}$ of the gluon plasma with initial conditions given by the pQCD calculation with the set MRSH [16]. Solid curve: ideal hydro flow with complete initial thermalization. Dashed curve: free streaming of the initial gluon system.

and expands adiabatically thereafter. Then we only need to compute the initial entropy and convert that into the final state multiplicity. Pressure, energy density and entropy density are $P_{g}=a T^{4}, \epsilon_{g}=3 a T^{4}, s_{g}=4 a T^{3}$. Again, we estimate the longitudinal size of the comoving volume as $\Delta z=\tau \Delta y$ and the energy density at the central rapidity region becomes $\epsilon_{g}\left(\tau_{i}\right)=\bar{E}_{T, g}^{P b P b}\left(|y| \leq 0.5, p_{0}\right) p_{0} /\left(\pi R_{P b}^{2}\right)$. The charged final multiplicity is obtained as

$$
\begin{aligned}
\frac{d N_{c h}}{d y} & \approx \frac{2}{3} \frac{1}{3.6} \frac{d S}{d y} \approx \frac{2}{3} \frac{1}{3.6} \pi R_{A}^{2} 4 a T_{i}^{3} \tau_{i} \\
& =\frac{2}{3} \frac{4}{3.6}\left[\frac{1}{27} \pi R_{A}^{2} a \tau_{i}\left\{\bar{E}_{T, g}^{P b P b}(|y|<0.5)\right\}^{3}\right]^{1 / 4} \\
& =914 \quad \mathrm{DO} 1 \\
& =1350 \quad \mathrm{D} 0^{\prime} \\
& =2180 \quad \mathrm{H}, \\
& =3360 \quad \mathrm{D}-^{\prime} .
\end{aligned}
$$

It is interesting to notice how crucial the lifetime $\tau_{c}$ of the (Q)GP-phase is for the high multiplicities. From Eq. (10) it is observed that due to entropy conservation

$$
\frac{d N_{c h}}{d y} \sim T_{i}^{3} \tau_{i}=T_{c}^{3} \tau_{c} \sim \tau_{c}
$$

since $T_{c}$ is in principle fixed by the theory. Therefore, an increase in the initial parton production at $\tau_{i}=0.1 \mathrm{fm}$ enhances the lower limit of the final multiplicity through increasing the lifetime of the plasma. 


\section{CONCLUSIONS}

I have discussed semihard transverse energy and parton production in the midrapidity unit in central $\mathrm{PbPb}$ collisions, and, considered initial conditions for early QGP formation at $\tau=0.1 \mathrm{fm}$ [13]. The main emphasis of the talk has been on the consequences of the new HERA parton distributions $[15,16]$ to minijet production at the small scales $p_{T} \sim 2 \mathrm{GeV}$. Especially, this lowest order pQCD calculation shows that the early QGP is clearly gluon dominated both at RHIC and LHC. For LHC, with the new, small- $x$ enhanced parton distributions it was also shown that rapid thermalization of the gluon system may be possible: initially at $\tau=0.1 \mathrm{fm}$ there are enough gluons produced so that $\epsilon_{g} / n_{g} \sim 2.7 T_{\mathrm{eq}}$ as in an ideal gas of massless bosons. However, the isotropization of the momenta what was not studied in detail [22].

Uncertainties in the pQCD calculation of the initial conditions were pointed out. Especially corrections due to shadowing, both in a proton and in a nucleus, were discussed, but not yet implemented. For RHIC it seems that the shadowing in a proton may be neglected but nuclear shadowing should be taken into account. For LHC, since probing smaller $x$ 's, one should include all shadowing effects simultaneously [30]. Clearly, in this sense, RHIC will be unaffected by the problems and uncertainties in the parton distributions at $x \lesssim 0.01$, whereas LHC will be affected by these complications. However, besides problems, the small- $x$ enhancement causes also larger initial energy and particle densities, resulting in longer lifetime for the QGP and larger multiplicities. A lower limit for multiplicites in the LHC nuclear collisions was also estimated [13]. When the forthcoming colliders are in operation, we will have a truly interesting sequence of experiments, AGS,SPS $\rightarrow$ RHIC $\rightarrow$ LHC to study, and a wide range of multiplicities to understand, hopefully in terms of a forming quark-gluon plasma.

\section{Acknowledgements}

I would like to thank K. Kajantie, V. Ruuskanen, M. Vänttinen and X.-N. Wang for helpful discussions.

\section{REFERENCES}

[1] T. K. Gaisser and F. Halzen, Phys. Rev. Lett. 54 (1985) 1754;

L. Durand and H. Pi, Phys. Rev. Lett. 58 (1987) 303;

G. Pancheri and Y. N. Srivastava, Phys. Lett. B182 (1986) 199.

[2] UA1 collaboration, C. Albajar et al., Nucl. Phys. B309 (1988) 405.

[3] K. Kajantie, P. V. Landshoff and J. Lindfors, Phys. Rev. Lett. 59 (1987) 2527.

[4] K.J. Eskola, K. Kajantie and J. Lindfors, Nucl. Phys. B323 (1989) 37.

[5] J.P. Blaizot, A.H. Mueller, Nucl. Phys. B289 (1987) 847.

[6] X.-N. Wang and M. Gyulassy, Phys. Rev. D44 (1991) 3501;

Phys. Rev. D45 (1992) 844; Phys. Rev. Lett. 68 (1992) 1480.

[7] K. Geiger and B. Müller, Nucl. Phys. B369 (1992) 600;

K. Geiger, Phys. Rev. D47 (1993) 133. 
[8] G. Gustafson, Nucl. Phys. A566 (1994) 233, in Proc. Quark Matter '93, eds. E. Stenlund, H.-A. Gustafsson, A. Oskarsson and I. Otterlund.

[9] K. Kajantie and T. Matsui, Phys. Lett. B164 (1985) 373;

G. Gatoff, A. K. Kerman and T. Matsui, Phys. Rev. D36 (1987) 114.

[10] K.J. Eskola and M. Gyulassy, Phys. Rev. C47 (1993) 2329.

[11] X.-N. Wang, Phys. Rev. D43 (1991) 104.

[12] T. Sjöstrand and M. van Zijl, Phys. Rev. D36 (1987) 2019;

T. Sjöstrand, Comp. Phys. Comm. 39 (1986) 347;

T. Sjöstrand and M. Bengtsson, ibid. 43 (1987) 367.

[13] K.J. Eskola, K. Kajantie and P. V. Ruuskanen, Phys. Lett. B332 (1994) 191.

[14] H1 Collaboration, I. Abt et al., Nucl. Phys. B407 (1993) 515;

ZEUS Collaboration, M. Derrick et al., Phys. Lett. B316 (1993) 412.

[15] A.D. Martin, W.J. Stirling and R.G. Roberts, Phys. Lett. B306 (1993) 145.

[16] A.D. Martin, W.J. Stirling and R.G. Roberts, RAL preprint 93-077 (1993).

[17] D. W. Duke and J. F. Owens, Phys. Rev. D30 (1984) 49.

[18] S. D. Ellis, Z. Kunszt and D. E. Soper, Phys. Rev. Lett. 62 (1989) 726; Phys. Rev. D40, (1989) 2188; Phys. Rev. Lett. 69 (1992) 1496;

Z. Kunszt and D. E. Soper, Phys. Rev. D46 (1992) 192.

[19] K. J. Eskola and X.-N. Wang "High $p_{T}$ Jet Production in $p p$ Collisions", Preprint HU-TFT-94-44, in Hard Processes in Hadronic Interactions, H. Satz and X.-N. Wang (eds.).

[20] A. Donnachie and P. V. Landshoff, Phys. Lett. B332 (1994) 433.

[21] J. D. Bjorken, Phys. Rev. D27 (1983) 140.

[22] K. J. Eskola and X.-N. Wang, Phys. Rev. D49 (1994) 1284.

[23] UA2 Collaboration, J. A. Appel et al., Phys. Lett. B 160 (1985) 349;

UA1 Collaboration, G. Arnison et al., Phys. Lett. B 172 (1986) 461;

UA1 Collaboration, C. Albajar et al., Nucl. Phys. B309 (1988) 405;

CDF Collaboration, F. Abe et al.,Phys. Rev. Lett. 62 (1989) 613.

[24] see Li Xiong's talk in this conference.

[25] S. J. Parke and T.R. Taylor; Phys. Rev. Lett. 56 (1986) 2459;

Z. Kunszt and W. J. Stirling; Phys. Rev. D37 (1988) 2439.

[26] V.N. Gribov and L.N. Lipatov, Sov. J. Nucl. Phys. 15 (1972) 438;

G. Altarelli and G. Parisi, Nucl. Phys. B126 (1977) 298;

Yu. L. Dokshitzer, Sov. Phys. JETP 46 (1977) 641.

[27] L. V. Gribov, E. M. Levin and M. G. Ryskin, Phys. Rep. 100 (1983) 1; Nucl. Phys. B188 (1981) 555; Zh. Eksp. Teor. Fiz. 80 (1981) 2132;

A. H. Mueller and J. Qiu, Nucl. Phys. B268 (1986) 427.

[28] J. Collins and J. Kwieciński, Nucl. Phys. B335 (1990) 89.

[29] J. Kwieciński, A. D. Martin, W. J. Stirling and R. G. Roberts, Phys. Rev. D42 (1990) 3645.

[30] K.J. Eskola, J. Qiu and X.-N. Wang, Phys. Rev. Lett. 72 (1994) 36.

[31] M. Arneodo, Phys. Rep. 240 (1994) 301. 
[32] see Raju Venugopalan's talk in this conference.

[33] EM Collaboration, M. Arneodo et al., Nucl. Phys. B333 (1990) 1;

NM Collaboration, P. Amaudruz et al, Z. Phys. C51 (1991) 387;

E665 Collaboration, M. R. Adams et al., Phys. Rev. Lett. 68 (1992) 3266.

[34] K.J. Eskola, Nucl. Phys. B400 (1993) 240.

[35] K.J.Eskola and X.-N. Wang, work in progress.

[36] A Letter of Intent for A Large Ion Collider Experiment (ALICE), CERN/LHCC/9316. 
